Documentation et bibliothèques

DOCUMENTATION BIBLIOTHËQUES

\title{
Les langages documentaires à l'école de bibliothéconomie et des sciences de Information (EBSI)
}

\section{Michèle Hudon}

Volume 52, numéro 2, avril-juin 2006

Les langages documentaires

URI : https://id.erudit.org/iderudit/1030021ar

DOI : https://doi.org/10.7202/1030021ar

Aller au sommaire du numéro

\section{Éditeur(s)}

Association pour l'avancement des sciences et des techniques de la documentation (ASTED)

\section{ISSN}

0315-2340 (imprimé)

2291-8949 (numérique)

Découvrir la revue

\section{Citer cet article}

Hudon, M. (2006). Les langages documentaires à l'école de bibliothéconomie et des sciences de Information (EBSI). Documentation et bibliothèques, 52(2),

161-162. https://doi.org/10.7202/1030021ar

Tous droits réservés (C) Association pour l'avancement des sciences et des techniques de la documentation (ASTED), 2006
Ce document est protégé par la loi sur le droit d'auteur. L'utilisation des services d'Érudit (y compris la reproduction) est assujettie à sa politique d'utilisation que vous pouvez consulter en ligne.

https://apropos.erudit.org/fr/usagers/politique-dutilisation/ 


\title{
Les langages documentaires à l'école de bibliothéconomie et des sciences de l'information (EBSI)
}

\author{
MICHÈLE HUDON \\ École de bibliothéconomie et des sciences de l'information \\ Université de Montréal \\ michele.hudon@umontreal.ca
}

\section{L} E PROGRAMME ACTUEL DE MAîtrise professionnelle en sciences de l'information offert à l'Université de Montréal a été implanté en 1998. Quatre options sont alors venues remplacer les neuf profils qui étaient offerts dans le programme précédent. Ces quatre options sont: Archivistique, Bibliothéconomie, Gestion électronique de l'information (GIE) et Gestion stratégique de l'information (GSI). Les options sont ouvertes aux étudiants de la deuxième année de la maîtrise ou, au plus tôt, lorsqu'une majorité des 9 cours du tronc commun ont été complétés et réussis. Au cours des discussions entourant la dernière révision du programme, c'est par consensus que le tronc commun a été établi : l'ensemble de la matière présentée durant la première année de la maîtrise, dans les 9 cours obligatoires du tronc commun, est considéré comme base de connaissances essentielles à tout professionnel de l'information, quels que soient le milieu dans lequel il sera appelé à travailler et les tâches qui lui seront dévolues. Le programme de maîtrise inclut également un stage obligatoire lié à l'option choisie par chacun des étudiants et défini en fonction de ses intérêts professionnels futurs ${ }^{1}$.

\section{Le tronc commun}

C'est dans le cours «Analyse et représentation documentaires $2^{2}$ » que les étudiants sont initiés aux opérations d'indexation, de condensation et de classification, ainsi qu'aux outils principaux qui permettent de les accomplir. Comme tous les autres cours du tronc commun, ce cours d'une valeur de 3 crédits (sur les 54 à compléter pour l'obtention du diplôme) exige des étudiants 42 heures de présence en classe, 14 heures de travail pratique supervisé en laboratoire et environ 79 heures de travail personnel sur des exercices qui seront évalués. Les heures de classe sont consacrées à des exposés théoriques sur les thématiques étudiées, soutenus par des exemples appropriés tirés de catalogues ou de bases de données existantes. Sauf

1. On trouvera une représentation de la structure du programme de maitrise en sciences de l'information sur le site Web de l'EBSI à l'adresse <http://www.ebsi. umontreal.ca/cours/index.html $>$.

2. Le cours «Analyse et représentation documentaire 1 » porte sur la description des ressources, sur la sélection des accès à la notice bibliographique (autres que les accès sujets), sur la gestion des fichiers d'autorité (autres que les fichiers d'autorité des sujets) et sur la gestion des catalogues. exception, aucune application pratique n'est faite en classe, le nombre d'étudiants dans le groupe (entre 75 et 9o) rendant difficilement applicable cette pratique pédagogique. Les exercices pratiques supervisés se font en groupes plus restreints de 25 à 30 étudiants, aux laboratoires de catalogage et d'informatique.

Depuis quelques années, la répartition des thématiques couvertes dans le cours obligatoire de première année, tant au niveau de la théorie que de la pratique, s'effectue de la façon suivante: 1) Introduction générale à l'analyse documentaire ( 3 heures de théorie); 2) Indexation en vocabulaire libre (3 heures de théorie, 2 heures de travaux pratiques supervisés); 3) Indexation en vocabulaire contrôlé (12 heures de théorie, 8 heures de travaux pratiques supervisés); 4) Condensation ( 6 heures de théorie, 2 heures de travaux pratiques supervisés); 5) Classification (12 heures de théorie, 4 heures de travaux pratiques supervisés) ; 6) Indexation, condensation et classification automatique ( 3 heures de théorie). Plusieurs langages documentaires sont examinés et utilisés par les étudiants, soit durant des travaux pratiques supervisés, soit durant la réalisation de travaux personnels. Dans la section du cours qui porte sur l'indexation en vocabulaire contrôlé, les répertoires de vedettesmatière de l'Université Laval (RVM) et de la Library of Congress (LCSH) sont étudiés en détail et utilisés par les étudiants pour compléter des exercices pratiques. Les thésaurus font également l'objet de deux séances de cours et les étudiants sont amenés à les utiliser d'abord dans un exercice d'indexation et, par la suite, à développer eux-mêmes une structure thésaurale simple. Dans la section du cours qui est consacrée à la classification, 2 cours théoriques de 3 heures chacun et un exercice de 2 heures portent sur la Classification décimale Dewey (CDD) (tables principales et tables de subdivisions 1 et 2); la Classification de la Library of Congress (LCC) reçoit exactement la même attention.

Les étudiants utilisent les versions Web de tous ces langages documentaires. À l'EBSI, seule la CDD est également encore utilisée sous sa forme imprimée. L'École dispose d'une trentaine d'exemplaires de la $21^{\mathrm{e}}$ édition en français de la CDD, ce qui permet à l'étudiant de mieux percevoir l'importance de la structure hiérarchique et de sa représentation dans un système de classification. 
Les langages documentaires sont également présentés, indirectement cette fois, dans la perspective du rôle qu'ils peuvent jouer à l'autre extrémité du système de transfert d'information dans un autre cours du tronc commun, «Recherche d'information». Les cours «Analyse documentaire 2 » et «Recherche d'information" sont offerts en parallèle pendant la même session, ce qui permet de familiariser les étudiants à la complexité, à l'efficacité et à la nécessité des langages documentaires.

\section{Deuxième année de la maîtrise: option Bibliothéconomie}

L'étudiant qui a choisi l'option Bibliothéconomie doit compléter 4 cours obligatoires et effectuer un stage dans un milieu approprié. Un des cours obligatoires de l'option a pour objectif principal l'approfondissement des notions de classification et d'indexation à l'aide de vedettes-matière vues par l'ensemble des étudiants dans le tronc commun. Au cours de ses 42 heures de présence en classe ou en laboratoire, et d'un minimum de 90 heures de travail personnel, l'étudiant inscrit au cours "Organisation des ressources d'information" devra résoudre des cas complexes de classification à l'aide de la CDD (tables principales et toutes les tables de subdivisions) et de la LCC, ainsi que des cas complexes de représentation thématique à l'aide de vedettes-matière développées à partir des LCSH et du RVM. D'autres schémas de classification encyclopédiques (Classification décimale universelle) ou spécialisés (Bibliothèque nationale de médecine, ICONCLASS, Plan de classification des collections de Parcs Canada, etc.), ainsi que la classification à facettes de Ranganathan sont également exposés de façon théorique aux étudiants. Six heures de théorie venant appuyer un travail de recherche fouillé sont consacrées aux structures hiérarchiques encyclopédiques ou spécialisées qui sont développées pour organiser les ressources du Web. Seules les versions Web de tous les langages documentaires, incluant WebDewey, sont exploitées dans ce cours.

\section{Deuxième année de la maîtrise: option Gestion d'information électronique}

Létudiant qui a choisi l'option Gestion d'information électronique (GIE) développe de son côté sa connaissance des thésaurus dans le cours «Analyse documentaire avancée». Des exercices appropriés permettent de mieux saisir l'utilité du thésaurus bien structuré pour l'indexation et la recherche d'information. L'étudiant est également appelé à développer un petit thésaurus spécialisé à l'aide d'un logiciel approprié, se préparant ainsi à une tâche dévolue au professionnel dans divers milieux documentaires.
Notons en passant qu'en plus des 4 cours obligatoires de son option, l'étudiant doit également s'inscrire à 3 cours supplémentaires. On observe au fil des années que plusieurs étudiants inscrits aux options Bibliothéconomie et GIE s'inscrivent simultanément aux 2 cours constituant une suite logique au cours "Analyse et représentation documentaires 2 ", soit «Organisation des ressources d'information» et "Analyse documentaire avancée». Offerts en parallèle pendant le même trimestre, ces deux cours ne sont jamais au même endroit dans la grille horaire.

\section{Les cours optionnels}

Une banque supplémentaire de cours optionnels est également disponible. Aucun cours n'y est consacré à l'application des langages documentaires. Ceux-ci sont toutefois évoqués de façon plutôt théorique dans les cours "Organisation des connaissances ", «Indexation et condensation automatiques » et «Recherche d'information avancée».

\section{Remarques supplémentaires}

Nous n'avons présenté ici que les cours au sein desquels les langages documentaires constituent un objet d'étude important. Il est évident qu'il est fait référence aux langages documentaires dans plusieurs autres cours du programme de maîtrise, souvent dans une terminologie différente ou dans une perspective bien particulière de recherche d'information, d'automatisation, d'application à la gestion des archives, etc.

Le lecteur constatera que l'importance (en nombre d'heures de théorie et de pratique) des langages documentaires dans le programme de formation de l'EBSI est bien moindre que celle qu'on leur consent dans les programmes de techniques de la documentation. À l'EBSI, l'accent est indéniablement mis sur la théorie et l'objectif est d'aider l'étudiant à percevoir l'importance des langages documentaires dans les systèmes automatisés de transfert d'information et à comprendre pourquoi les langages documentaires sont structurés comme ils le sont, plutôt que sur l'exploration de techniques d'utilisation. Les étudiants ne sont pas nécessairement heureux de cette situation et déplorent souvent le peu de temps consacré à la pratique. À sa sortie de l'EBSI, le diplômé n'est pas encore un spécialiste de l'indexation, de la classification et de la gestion de langages documentaires. Il possède cependant des connaissances de base solides qui lui serviront de tremplin pour le développement de compétences soit par la pratique quotidienne de l'analyse documentaire, soit par la supervision d'analystes, de techniciens ou de professionnels, et par l'étude des cas complexes qui lui seront soumis par ces derniers. $\odot$ 